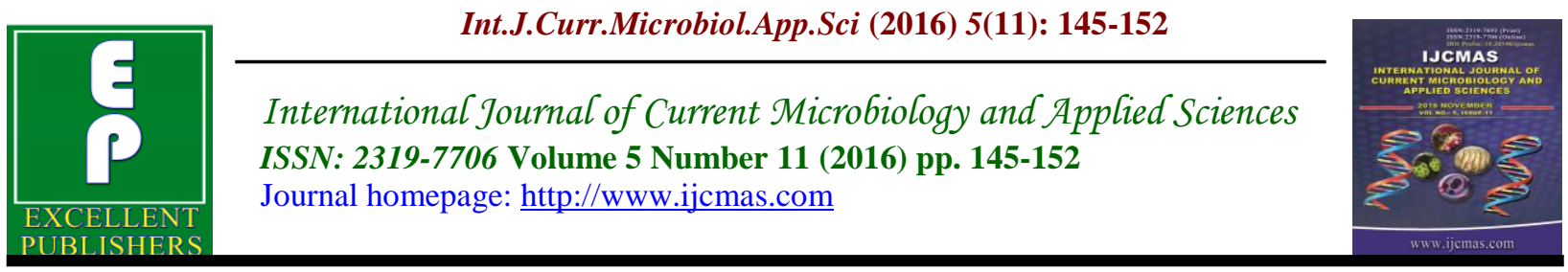

Original Research Article

http://dx.doi.org/10.20546/ijcmas.2016.511.017

\title{
Comparative Analysis of Antibacterial Activity of Aqueous, Ethanolic, Methanolic and Acetone Extracts of Commercial Green Tea and Black Tea against Standard Bacterial Strains
}

\author{
Abhishek Mehta ${ }^{1 *}$, Gaurav Saxena $^{2}$ and Abin Mani ${ }^{3}$ \\ ${ }^{1}$ Department of Microbiology, K.D. Medical College, Hospital\& Research Center, Mathura, (Ex- \\ Assistant Professor, Department of Microbiology, Peoples College of Medical Sciences \& \\ Research Centre, Bhopal), India \\ ${ }^{2}$ Department of Microbiology, People's College of Medical Sciences, Bhopal, India \\ ${ }^{3}$ Senior Scientist, Center for Scientific Research \& Development, \\ People's University, Bhopal, India \\ *Corresponding author
}

Keywords

Agar well diffusion, Anti bacterial activity, Antibiotic resistance, Black tea, Catechins, Green tea, Inhibition zone diameter.

\section{Article Info}

Accepted:

12 October 2016

Available Online:

10 November 2016

\section{A B S T R A C T}

Green tea and Black tea extracts are known to exhibit significant antibacterial activity against many pathogenic microbes. With the emerging threat of growing antibiotic resistance amongst bacterial strains posing serious challenge to clinicians left with few options there has been an increasingly growing interests to explore new antimicrobial compounds from herbal extracts, which could be incorporated in the treatment of such infections. So, this study was undertaken to evaluate and compare the antibacterial activity of different extracts of Green tea and Black tea against standard bacterial strains. This Cross-sectional Analytical study was conducted in the Department of Microbiology of a Tertiary care Teaching hospital of Central India over a period of three months. Antimicrobial activity of Aqueous, Ethanolic, Methanolic and Acetone extracts of Green tea and Black tea was determined by Agar well diffusion method. Green tea extracts exhibited higher antimicrobial activity than Black tea extracts. Amongst Green tea extracts aqueous extract was found to be most effective while amongst Black tea extracts methanolic extract was most effective. All tea extracts have shown significant antibacterial activity against S.aureus ATCC 25922 with Aqueous extract of Green tea exhibiting highest activity. All Green tea extracts exhibited significant activity against E.coli ATCC 25923 higher than Black tea extracts. As compared to Green tea extracts, Black tea extracts showed much lower activity against P.aeruginosa ATCC 27853. S.aureus was found to be most susceptible to tea extracts followed by E.coli and P.aeruginosa. Green tea and Black tea extracts have shown significant antibacterial activity with former being more effective than later. In future there is immense potential of clinical application of polyphenolic contents of tea extracts as adjuvant therapeutic agents to tackle the menace of growing antibiotic resistance. 


\section{Introduction}

Tea prepared from the leaves of the plant Camellia sinensis is very well known as the most widely consumed beverage in the world. Green tea and Black tea are the two main types of tea products. Tea extracts from both types exhibit significant antibacterial activity against many pathogenic microbes mainly attributed to the polyphenolic fractions esp. Catechins which primarily include Epigallocatechin-gallate (EGCG), Epigallocatechin (EGC), Epicatechin gallate (ECG) and Epicatechin(EC) (Mbuthia et al., 2014; Archana et al., 2011; Radji et al., 2013).

There is an emerging threat to public health due to growing antibiotic resistance amongst bacterial pathogens triggered by malpractices involving excessive, inappropriate and injudicious use of antibiotics. To combat the emergence and spread of multi drug resistant pathogens there is a growing need to explore newer antimicrobial compounds particularly from herbal extracts which could be incorporated as adjuvant therapeutic agents in the treatment of such life threatening infections (Mbuthia et al., 2014). In India very few comparative studies are conducted to evaluate antibacterial property of various tea extracts. Keeping this in mind we had undertaken this cross sectional analytical study involving comparative evaluation of antibacterial activity of aqueous, ethanolic, methanolic and acetone extracts of Commercial Green tea and Black tea against Standard (ATCC) bacterial strains.

\section{Materials and Methods}

This cross sectional Analytical study was conducted in the Dept. of Microbiology of a Tertiary care Teaching Hospital of Central India from August 2015 to October 2015.
The comparative evaluation of antibacterial activity of Aqueous, Ethanolic, Methanolic and Acetone extracts of Commercial Green Tea and Black Dust Tea was done against three Standard (ATCC) bacterial strains:

1)Staphylococcus aureus ATCC 25923

2)Escherichia coli ATCC 25922

3)Pseudomonas aeruginosa ATCC 27853

By Agar well Diffusion method.

Preparation of Tea extracts by Cold extraction method: (Archana et al., 2011; Kumar et al., 2012)

Dried leaves of Green tea plant Camellia sinensis (Goodricke Barnesbeg Darjeeling Green tea) and Black tea procured from local vendor and pulverized to coarse powder.

Both forms are processed separately by Cold Extraction method.

1)Aqueous Extract: $10 \mathrm{gms}$. Of the tea powder was soaked in $100 \mathrm{ml}$.of sterile distilled water for 2 consecutive days with intermittent shaking at room temperature. After maceration it was strained through a sterile muslin cloth and finally filtered through sterile Whatman No.1 filter paper.The filtrates were then concentrated under reduced pressure using Rotary Vaccum Evaporator (Equitron-Roteva) at $40^{\circ} \mathrm{C}$. The Concentrate (semisolid paste) was then allowed to dry at room temperature under aseptic conditions. The dried crude extract was stored in sterile air tight labelled bottles at $4^{0} \mathrm{C}$ until use.

2)Solvent Extracts (Ethanolic / Methanolic /Acetone Extracts): 10Gms.of the tea powder soaked separately in $100 \mathrm{ml}$ of $95 \%$ Ethanol, $70 \%$ Methanol and 100\% Acetone and allowed to macerate at room temperature for 5days with occasional 
shaking. Then, strained through sterile muslin cloth and finally filtered through sterile Whatman No.1 filter paper. The filtrates were then concentrated under reduced pressure using Rotary Vaccum Evaporator at $40^{\circ} \mathrm{C}$.The solvents are completely removed by evaporation to yield dried extract powder stored separately in sterile, airtight labeled bottles at $4^{0} \mathrm{C}$ until use.

Antibacterial Activity Test (Mbuthia et al., 2014; Archana et al., 2011; Tariq et al., 2013)

The dry powder of each crude extract is weighed on digital balance and dissolved in $0.1 \%$ DMSO (Dimethyl Sulfoxide) separately to prepare 5 concentrations of each extract $(200,100,50,25,12.5 \mathrm{mg} / \mathrm{ml})$ and stored in sterile labelled aliquots in freezer tray. The antibacterial activity of different concentrations of Aqueous, methanolic, ethanolic and Acetone extracts each of both Commercial green tea and Black tea is assessed separately by Agar Well Diffusion method against Standard bacterial strains (E.coli ATCC 25922, S.aureus ATCC 25923 and P.aeruginosa ATCC 27853).

Test organisms are subcultured in sterile peptone water, turbidity matching $0.5 \mathrm{Mac}$ Farland standard and inoculated on Muller Hinton Agar plates so as to achieve a uniform lawn culture. Six wells(5wells at the periphery and one at the centre) of $6 \mathrm{~mm}$.diameter each were aseptically punched on MHA plates using sterile Cork borer keeping a uniform minimum distance of $30 \mathrm{~mm}$ between adjacent wells. The five wells at the periphery were loaded with $50 \mu 1$. each of the specific tea extract at different concentrations .Central well is loaded with $50 \mu \mathrm{l}$ of DMSO serves as Negative control.
After $24 \mathrm{hrs}$ of incubation in air at $37^{\circ} \mathrm{C}$, the plates were taken out and observed for appearance of Zones of inhibition around the wells. The antibacterial activity of the crude tea extracts at different concentrations is evaluated by measuring Zone size diameter in $\mathrm{mm}$.

\section{Results and Discussion}

This study was undertaken to evaluate and compare the antibacterial activity of different extracts of Green tea and Black tea against Standard ATCC bacterial strains by Agar well diffusion method.

The assessment of the antibacterial activity of different extracts in this study was based on measurement of diameter of Zones of inhibition formed around the charged wells as depicted in Tables 1,2 ;Figures 1,2 \& Pictures 1,2,3.

S.aureus was found to be most susceptible to tea extracts followed by E.coli and P.aeruginosa

Amongst all tea extracts tested the Aqueous extract of Green tea has shown the highest antimicrobial activity against S.aureus ATCC 25922.

But all other extracts of Green tea has also shown a significant and modest antibacterial activity against S.aureus except Methanolic extract which exhibited poor or absent activity at lower concentrations.

As compared to Aqueous extract of Green tea, all black tea extracts has shown lower but still significant antimicrobial activity except at lower concentrations.

While assessing the antimicrobial activity of Green tea extracts against E.coli ATCC 25923 we found that all the four Green tea 
extracts exhibited almost equal and significant activity at respective concentrations. But solvent extracts have shown poor or absent activity at low concentration of $12.5 \mathrm{mg} / \mathrm{ml}$.

As compared to Green tea extracts, Black tea extracts exhibited lower antimicrobial activity against E.coli. Amongst all extracts of black tea, Methanolic extracts showed highest antimicrobial activity at higher concentrations. All extracts of Black tea exhibited poor or absent activity at lower concentrations.

While examining the antibacterial activity of Green tea extracts against P.aeruginosa ATCC 27853, Aqueous extract exhibited highest antimicrobial activity followed by methanolic, ethanolic and acetone extracts. Acetone extract showing poor or no activity at lower concentrations.

As compared to Green tea extracts,Black tea extracts exhibited much lower antimicrobial activity against P.aeruginosa. Methanolic extracts showed highest antimicrobial activity. Rest of the extracts showing poor or no activity at lower concentrations.

\section{Discussion}

It is clearly evident from this study that all the four extracts of Green tea and Black tea do exhibit remarkable antibacterial activity which vary with the type of extract, its concentration and also with the target bacterial strain.

The antibacterial property of tea extracts is mainly attributed to their polyphenolic components mainly the simple isoflavanoids (Catechins) (Mbuthia et al., 2014; Radji et al., 2013; Kumar et al., 2012). The primary difference between Green tea and Black tea lies in their processing. Black tea is prepared by a postharvest auto-oxidation (fermentation) catalyzed by polyphenol oxidase. While in case of Green tea leaves are not subjected to fermentation but instead steamed after drying to inactivate the polyphenol oxidase enzyme. The variation in polyphenolic composition of different tea products is due to this difference in processing. During Black tea processing the auto-oxidation of tea leaves convert catechins to more complex Theaflavins(TFs) and Thearubigins(TRs) (Koech et al., 2013; Hamilton Miller et al., 1995; Hossain et al.,2014).

That is why Green teas has significantly higher Catechin content than black teas. This explains for the higher antimicrobial activity of Green tea extracts as compared to Black tea extracts.

As shown by this study S.aureus is much more susceptible to tea extracts as compared to E.coli and P.aeruginosa. This is due to much greater binding of negatively charged EGCG to the positively charged lipid bilayer constituent of cell membrane of Gram positive bacteria. The bactericidal effect of EGCG is attributed to membrane perturbation (Koech et al., 2013; Hossain et al., 2014).

Apart from catechins the other tea flavanols like Quercitin, Kaempferol and Myricetin were also found to have antibacterial activity against Gram positive bacteria at varying concentrations. Quercitin exhibits remarkable activity against S.aureus even higher than EGCG (Hamilton Miller et al., 1995).

Amongst 4000 bioactive compounds present in leaves of the plant Camellia sinensis there are around 100 compounds which have been found to have antimicrobial activity against bacterial pathogens to varying extents at different concentrations. 
Amongst these compounds the antimicrobial activity of tea extracts is mainly attributed to catechins particularly EGCG and ECG. The concentration of these catechins in tea extracts varies not only with the type of tea (Green tea, Black tea, White tea, purple tea,
Olong tea, Kombucha tea) determined by difference in their post harvest processing but also varies with the method of extraction process involved in preparing tea extracts (Cold extraction/Hot extraction, Aqueous extraction/Solvent extraction).

Table.1 Antibacterial activity of Green tea extracts against Standard Bacterial strains

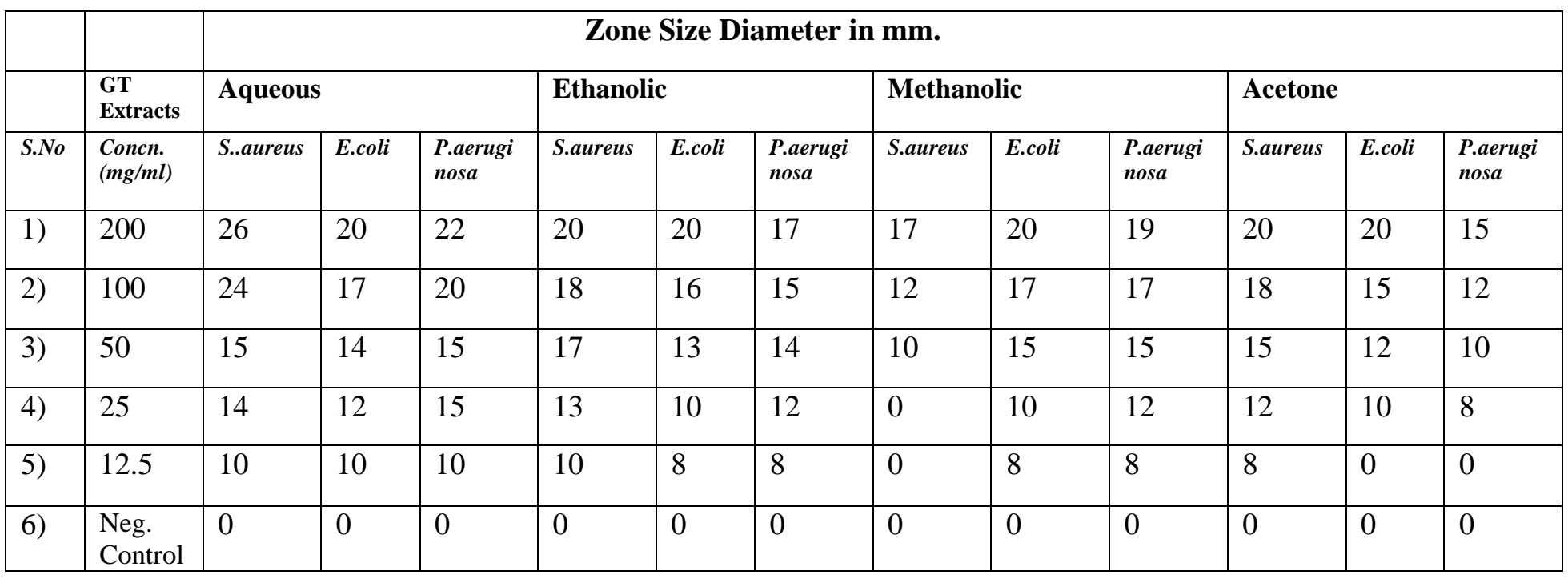

Table.2 Antibacterial activity of Black tea extracts against Standard Bacterial strains

\begin{tabular}{|c|c|c|c|c|c|c|c|c|c|c|c|c|c|}
\hline \multirow[b]{3}{*}{ S.No } & \multirow{3}{*}{$\begin{array}{l}\begin{array}{l}\text { GT } \\
\text { Extract }\end{array} \\
\begin{array}{l}\text { Concn. } \\
(m g / m l)\end{array}\end{array}$} & \multicolumn{12}{|c|}{ Zone Size Diameter in $\mathbf{m m}}$. \\
\hline & & \multicolumn{3}{|c|}{ Aqueous } & \multicolumn{3}{|c|}{ Ethanolic } & \multicolumn{3}{|c|}{ Methanolic } & \multicolumn{3}{|c|}{ Acetone } \\
\hline & & S..aureus & E.coli & P.aeruginosa & S.aureus & E.coli & P.aeruginosa & S.aureus & E.coli & P.aeruginosa & S..aureus & E.coli & P.aeruginosa \\
\hline 1) & 200 & 20 & 13 & 10 & 17 & 15 & 13 & 20 & 17 & 15 & 16 & 14 & 12 \\
\hline 2) & 100 & 18 & 12 & 10 & 15 & 14 & 11 & 17 & 15 & 12 & 15 & 12 & 12 \\
\hline 3) & 50 & 14 & 10 & 8 & 12 & 11 & 8 & 15 & 10 & 10 & 12 & 10 & 10 \\
\hline 4) & 25 & 10 & 8 & 0 & 10 & 9 & 0 & 12 & 8 & 9 & 11 & 8 & 8 \\
\hline 5) & 12.5 & 8 & 0 & 0 & 8 & 0 & 0 & 10 & 7 & 8 & 9 & 8 & 0 \\
\hline 6) & Neg.C & 0 & 0 & 0 & 0 & 0 & 0 & 0 & 0 & 0 & 0 & 0 & 0 \\
\hline
\end{tabular}


Fig.1 Antibacterial activity of Green tea extracts against Standard Bacterial strains

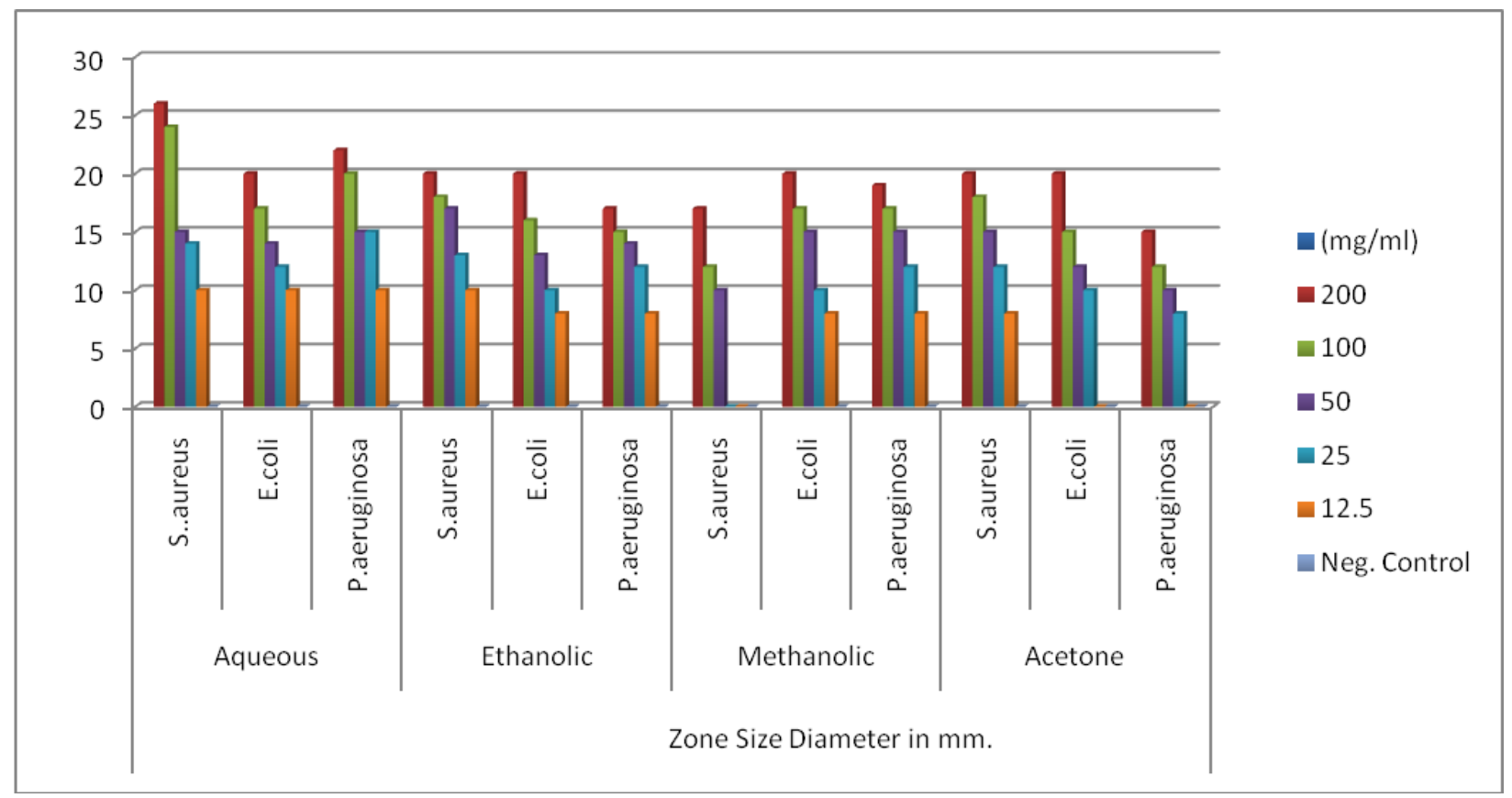

Fig.2 Antibacterial activity of Black tea extracts against Standard Bacterial strains

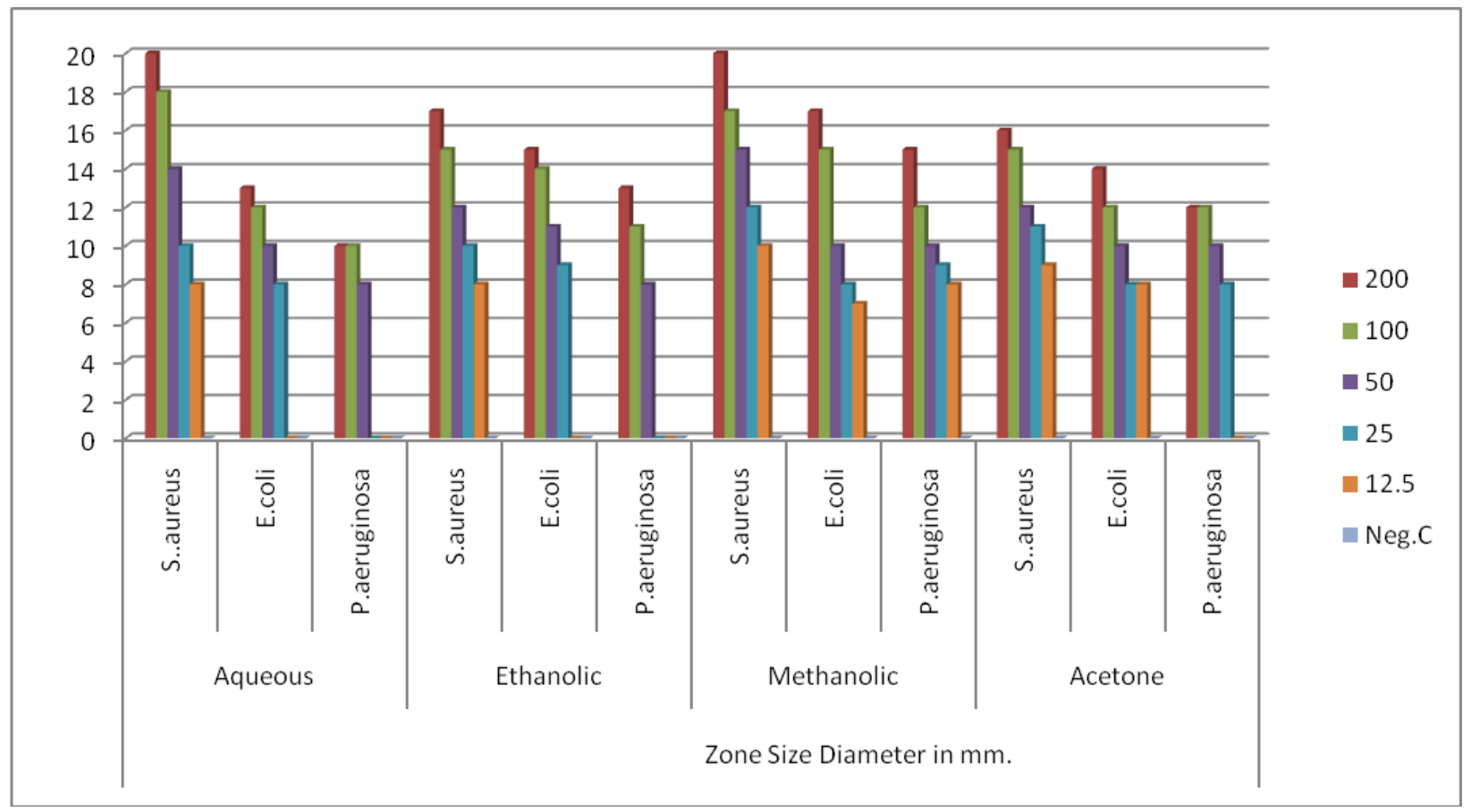


Photo.1 Antibacterial activity of Green tea extracts against P.aeruginosa ATCC 27853

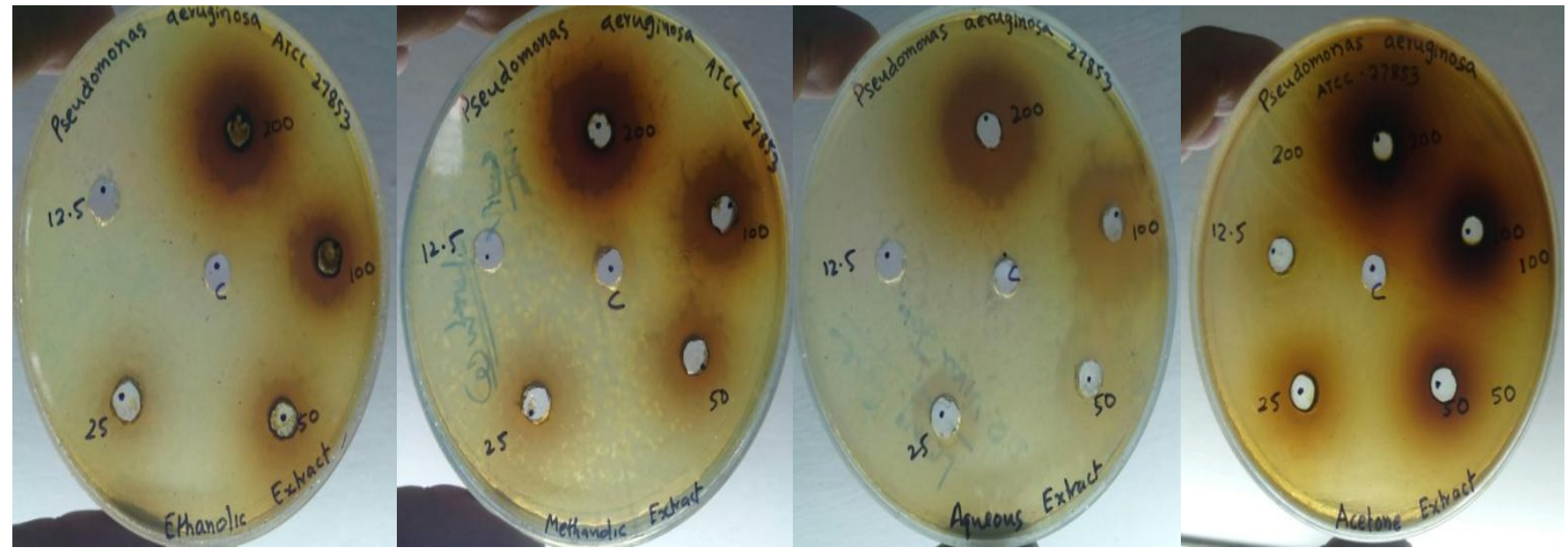

Photo.2 Antibacterial activity of Black-tea extracts against P.aeruginosa ATCC 27853
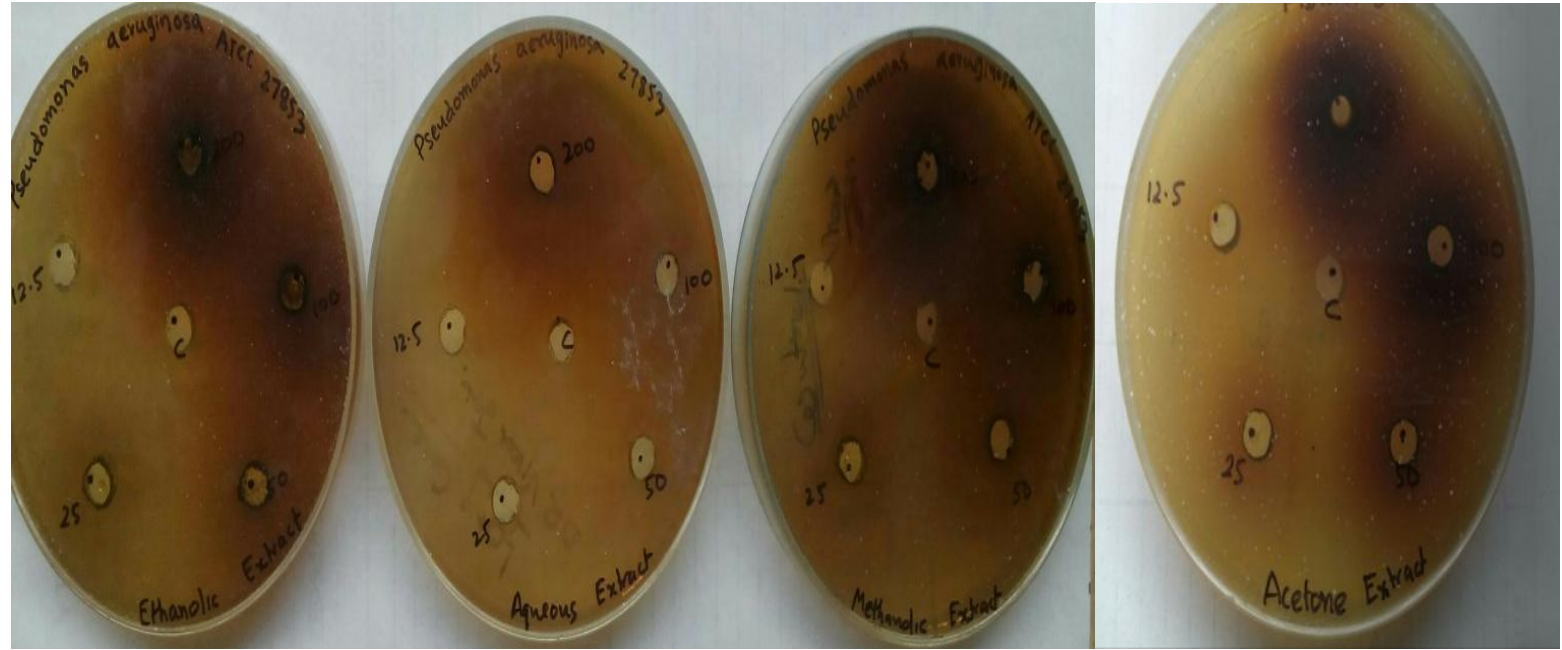

So, there is immense scope of research in this field for the exploration of newer bioactive compounds exhibiting antimicrobial activity in tea extracts and designing and improvising new better techniques of processing the tea products for the efficient extraction of such compounds which could be incorporated in near future as adjuvant therapeutic agents in the treatment of multi drug resistant infections in community as well as in hospital settings.

In conclusion, this study like many other in- vitro studies conducted throughout the world had proved, reconfirmed and consolidated the fact that tea extracts do have significant antimicrobial activity at varying concentrations against different bacterial pathogens. This study in addition had also shown that this antibacterial activity is dependent not only on the type of the tea product but also on different processing techniques involved in preparing tea extracts which determines the concentration of polyphenols particularly catechins responsible for this activity. 


\section{References}

Archana, S., Abraham, J. 2011. Comparative Analysis of antimicrobial activity of leaf extracts from fresh greentea,commercial green tea and black tea on pathogens. J. App. Pharma. Sci., 01(08): 149-152.

Hamilton-Miller, J.M.T. 1995. Antimicrobial Properties of Tea (Camellia sinensis L.) Antimicrobial Agents and Chemotherapy, 39(11): 2375-7.

Hossain, M.S., Nibir, Y.M., Zerin, S., Ahsan, N. 2014. Antibacterial Activities of the Methanolic Extract of Bangladeshi Black Tea against Various Human Pathogens. Dhaka Univ. J. Pharm. Sci., 13(1): 97-103.

Kumar, A., Thakur, P., Patil, S., Chandani, P. 2012. Antibacterial activity of green tea (Camellia sinensis) extracts against various bacteria isolated from environmental sources. Recent Res. Sci. Tech., 4(1): 19-23.

Koech, K.R., Wachira, F.N., Ngure, R.M., Wanyoko, J.K., Bii, C. and Karori, S.M. 2013. Antibacterial and Synergistic Activity of Different Tea Crude Extracts against Antibiotic Resistant S. Aureus, E. Coli and a Clinical Isolate of S. typhi. Sci. J. Microbiol., Volume 2013, Article ID: sjmb-115, 9 Pages, 2013. doi: $10.7237 / \mathrm{sjmb} / 115$.

Mbuthia, S.K., Wacharia, F.N., Koech, R.K. 2014. In vitro antimicrobial and synergistic properties of water soluble green and black tea extracts. African $J$. Microbiol. Res., 8(14):1527-153.

Patil, M.P., Patil, K.T., Ngabire, D., Seo, Y.B., Kim, G.D. 2016. Phytochemical, Antioxidant and Antibacterial Activity of Black Tea (Camellia sinensis). Int. J. Pharmacognosy and Phytochemical Res., 8(2); 341-346.

Radji, M., Agustama, R.A., Elya, B., Tjampakasari, $\quad$ C.R. 2013. Antimicrobial Activity of Green Tea Extract Against Isolates of Methicillin Resistant Staphylococcus aureus and Multi drug Resistant Pseudomonas aeruginosa. Asian Pac. J. Trop. Biomed., (8): 663-667.

Tariq, A.L., Reyaz, A.L. 2013. Camellia sinensis leaves a new treamtnet against Urinary Tract Infection caused by Pseudomonas fluorescens and Serratia sp. Int. J. Pharm. Sci. Res., 4(3): 154650.

Vazir, J., Inamdar, P., Desai, S., Patel, D., Meshram, D. 2014. Phytochemical Screening and in Vitro Antibacterial Activity of Camellia sinensis. Indo American J. Pharm. Res., 4(02): 115862.

\section{How to cite this article:}

Abhishek Mehta, Gaurav Saxena and Abin Mani. 2016. Comparative Analysis of Antibacterial Activity of Aqueous, Ethanolic, Methanolic and Acetone Extracts of Commercial Green Tea and Black Tea against Standard Bacterial Strains. Int.J.Curr.Microbiol.App.Sci. 5(11): 145152. doi: http://dx.doi.org/10.20546/ijcmas.2016.511.017 